\title{
Schistosomiasis prevalence and low-cost diagnostics in rural Northwestern Madagascar: a pilot study
}

\author{
Kyle E. Robinson ${ }^{1}$, Eric P. Grewal, MMSc${ }^{2}$, Bobbi S. Pritt, MD³ ${ }^{3}$ Margaret Lloyd ${ }^{4}$, Adriantiano M. Stephano ${ }^{4}$, Mamantsara \\ Fardine ${ }^{4}$, Lisa M. Brumble, MD ${ }^{5}$, Claudia R. Libertin, MD $^{5}$ \\ 1 Division of Infectious Diseases Mayo Clinic, Jacksonville, Florida, USA; Mada Clinics, Mavenitbao, Madagascar; Mayo Clinic Alix School of Medicine, \\ Rochester, Minnesota, USA, 2 Mada Clinics, Mavenitbao, Madagascar; Mayo Clinic Alix School of Medicine, Rochester, Minnesota, USA, ${ }^{3}$ Department \\ of Laboratory Medicine and Pathology, Mayo Clinic, Rochester, Minnesota, USA, ${ }^{4}$ Mada Clinics, Mavenitbao, Madagascar, ${ }^{5}$ Division of Infectious \\ Diseases Mayo Clinic, Jacksonville, Florida, USA \\ Keywords: low cost, parasitology, urine microscopy, madagascar, schistosomiasis \\ https://doi.org/10.29392/001c.22123
}

\section{Journal of Global Health Reports}

Vol. 5, 2021

\begin{abstract}
Background
Schistosomiasis is a parasitic disease that affects the health of hundreds of millions of people worldwide. Affordable diagnostic methods are necessary for developing countries to monitor schistosomiasis rates and treat infected individuals, particularly in, Madagascar, a country with the world's fifth highest rate of schistosomiasis.
\end{abstract}

\section{Methods}

We established the prevalence rate of schistosomiasis among the local population served by a clinic in rural northwestern Madagascar. Additionally, we compared different low-cost methods for schistosomiasis detection, including empirical diagnostic methods in use before this study. 500 patients were recruited in this study, of whom 380 received three tests-urine microscopy, point-of-care circulating cathodic antigen (POC-CCA) testing, and urinalysis to screen for Schistosoma haematobium and Schistosoma mansoni.

\section{Results}

By combining these modalities, we recorded a schistosomiasis prevalence rate of $64.47 \%$, which was significantly higher than the $4.84 \%$ of patients who were diagnosed and treated through previous empirical methods.

\section{Conclusions}

Our results indicate that screening tests are a necessary component of schistosomiasis control programs. The clinic continued to use urinalysis strip testing and urine microscopy to detect schistosomiasis after this study and decided to discontinue the use of POC-CCA S. mansoni urine testing because of cost. Increased awareness of schistosomiasis resulting from this screening program led to the installation of multiple infrastructure projects in local communities to improve access to potable water, demonstrating community benefits of schistosomiasis screening that extend beyond the identification of infected individuals.

Worldwide, more than 700 million people live in areas endemic to schistosomiasis, a parasitic disease caused by flatworms of the genus Schistosoma. ${ }^{1,2}$ Most of the human infections are caused by Schistosoma haematobium, Schistosoma mansoni, and Schistosoma japonicum. Symptoms depend on the infecting species. S. haematobium resides in the venous plexus surrounding the bladder; the other 2 species reside in the mesenteric plexus. Eggs are produced by adult female flatworms, which can propagate in blood vessels leading to nearby organs. Short-term complications of schistosomiasis include hematuria, hematochezia, diarrhea, and abdominal pain, although many cases may initially be asymptomatic. Long-term sequelae of schistosomiasis are the most worrisome and include hepatosplenomegaly, portal hypertension, hepatic fibrosis, bladder cancer, genital lesions, and renal failure. More than 250 million people are believed to be affected by this illness, the complications of which could contribute to as many as 200,000 deaths each year. ${ }^{2,3}$

Schistosomiasis is spread when infected persons pass Schistosoma spp eggs in urine or feces and this waste enters sources of freshwater used for bathing, cleaning, or recreation. The parasite matures in snail hosts before subsequent release of microscopic swimming parasitic forms (cercariae) that are capable of penetrating intact skin. This life cycle can be disrupted if water sources are isolated from either snails or human and animal waste.

Infections are commonly diagnosed through identifica- 
tion of eggs in centrifuged urine (S. haematobium) or stool (S. mansoni and $S$. japonicum) with light microscopy. Alternatively, antibodies to the Schistosoma parasite can be detected with commercially available assays. Infected persons can be treated with the antiparasitic drug praziquantel, which may be administered at the WHO recommendation of as a single dose $(40 \mathrm{mg} / \mathrm{kg}){ }^{4}$ Unfortunately, reinfection is common with ongoing exposure to contaminated water sources. However, repeated treatment (eg, annual mass drug administration to a susceptible population) greatly lowers the accumulated burden of disease, which tends to accrue after a lifetime of recurring exposure to cercariae. $^{5}$

Numerous countries have implemented programs to reduce the rate of schistosomiasis. ${ }^{6}$ Before discovery of praziquantel, early efforts used molluscicides to control the snail population. More recent programs have shifted to treatment of infected individuals, to education, and to provision of potable water sources. $S$. mansoni prevalence in Brazil decreased from $23 \%$ to $6 \%$ through community-wide, annual mass drug administration and water sanitation. ${ }^{6}$ Another program, in Morocco, decreased the prevalence of $S$. haematobium from $6.2 \%$ to $0.0 \%$ (no reported cases) through education and annual screening. Of note, Japan successfully eliminated $S$ japonicum before the advent of praziquantel by lining water sources with concrete and deploying a schoolbased treatment and education program. ${ }^{6}$

Madagascar is an East African island nation of 24 million people, $52.1 \%$ of whom are estimated to be infected with schistosomiasis. This represents the fifth-highest rate in the world. ${ }^{6}$ Political turmoil, including a 2009 coup d'état, has hampered efforts to control schistosomiasis rates. Only $11 \%$ of the population has access to improved sanitation, ${ }^{7}$ and $44 \%$ practices open defecation. ${ }^{8}$ In addition, education and understanding of schistosomiasis among the population remain poor, with many persons believing it is a sexually transmitted infection. ${ }^{9}$ Access to health care for much of the rural Malagasy population is limited to governmentrun basic health care centers, many of which lack physicians, microscopes, and laboratory testing for schistosomiasis. Discovering and implementing effective, sustainable, low-cost methods of schistosomiasis control and prevention are imperative to the health of the Malagasy population and those living in developing countries across the globe.

In the present study, we implemented a screening and treatment regimen for patients with schistosomiasis in rural northwestern Madagascar, a region endemic to $S$. mansoni and $S$. haematobium. ${ }^{10}$ Previously, the main clinic in the region diagnosed and treated schistosomiasis using empirical methods. Due to the lack of formal laboratory resources and training, empirical diagnosis was based largely on the presenting symptom of gross hematuria. Prior to this study, a review of previous health records from 600 patients indicated a preexisting diagnosis and treatment rate of $4.84 \%$ for schistosomiasis. The objective of this study was to establish the prevalence rate of schistosomiasis among the local population served by the clinic and to compare different methods for schistosomiasis detection, including the empirical diagnostic methods used by the clinic before this study.

\section{METHODS}

\section{PATIENTS AND STUDY CHARACTERISTICS}

This study was conducted at Mada Clinics, a rural nonprofit care facility located approximately $100 \mathrm{~km}$ from the nearest hospital in Antsiranana. At the time of the study, the clinic consisted of a 2-room adobe building that lacked electricity or running water and provided care for an estimated population of 15,000 villagers.

Between June 14 and July 15, 2019, all patients presenting for routine care were asked to provide a urine sample for schistosomiasis screening with microscopy, urinalysis strip, and urine antigen test. Participants were asked their village of residence, age, and sex for epidemiologic data collection. Participants were assigned a number for use in data records and samples to protect confidentiality. This study was approved by the Mayo Clinic Institutional Review Board in Rochester, Minnesota, and the Malagasy Ministry of Health in Antsiranana. Verbal consent was acquired from patients before study admission, including parental consent for minors. All patients were provided verbal education explaining schistosomiasis and its transmission route, to ensure informed consent.

\section{SAMPLE COLLECTION, PREPARATION, AND MICROSCOPY}

To establish a standard diagnosis of $S$. haematobium, urine centrifugation followed by microscopy was used as described previously. ${ }^{11}$ In brief, fresh urine samples were collected and centrifuged for 10 minutes with use of Eisco Labs Hand Crank Centrifuge, 4 Tube Capacity (Eisco Scientific LLC). The supernatant was decanted, and the remaining pellet was resuspended and placed on a $25.4 \times 76.2 \times 1.0-\mathrm{mm}$ glass slide. Alternatively, gravity sedimentation was used for 41 urine samples for comparison with centrifugation. Gravity samples were poured into $50-\mathrm{mL}$ tubes and left to settle for 24 hours. Subsequently, a pipette was inserted to the bottommost point of the tube, sediment withdrawn, and 2 drops of urine sediment placed on a glass slide. To reduce interobserver variability, a single trained microscopist scanned slides prepared with either method for S. haematobium eggs, using a compound light microscope at $400 \times$ magnification.

\section{URINE ANTIGEN TESTING AND URINALYSIS}

A commercial immunochromatographic lateral flow pointof-care (POC) test was used to test the urine for the presence of Schistosoma spp circulating cathodic antigen (CCA) (Schisto POC-CCA; Rapid Medical Diagnostics). The POCCCA test is capable of detecting antigens of $S$. mansoni and $S$. haematobium, although the sensitivity is greater for the former species. ${ }^{12}$ This assay is primarily intended to detect $S$. mansoni, but data were also collected for patients with confirmed $S$. haematobium infection to provide additional information about the utility of this test to detect either species. The test was performed in accordance with manufacturer instructions. Briefly, 2 drops of urine were extracted from urine samples before centrifugation, placed on the test cassette, and allowed to develop for 20 minutes. To assess for hematuria, a urinalysis reagent strip (Mission; 
ACON Laboratories, Inc) was quickly submerged in the urine sample and allowed to develop for 1 minute in accordance with the kit instructions. Results were recorded for both tests. The sensitivity of $S$. haematobium detection through urinalysis was determined by comparing results against the findings of urine microscopy, the established gold standard. ${ }^{11}$ Sensitivity values were calculated as previously described. ${ }^{13}$

\section{DIAGNOSIS AND TREATMENT}

All samples with visible eggs on microscopy were considered positive on the basis of the high specificity of microscopy for $S$. haematobium detection. ${ }^{14}$ All samples with positive POC-CCA test results were considered positive for schistosomiasis (caused by either $S$. haematobium or $S$. mansoni) on the basis of the previously reported high specificity of this test. ${ }^{12}$ Results of the POC-CCA test were reported as positive or negative, according to manufacturer instruction, after interpretation by a single, standardized observer. To observe a significant difference of at least 5\% in schistosomiasis prevalence between groups with a power of $80 \%$, we determined that a minimum sample size of 180 was necessary. For this study, the presence of blood on the urinalysis test strip was considered empirical evidence of schistosomiasis for all male patients without an otherwise likely clinical explanation (i.e. benign prostatic hyperplasia) and all female patients younger than 8 years or older than 60 years who did not have an otherwise likely clinical explanation.

Female patients between age 8 and 60 years were asked the date of their last menstrual cycle. ${ }^{14}$ If more than 1 month had passed, they were provided a pregnancy test. If pregnancy test results were negative, the patients were treated if they had a positive result on microscopy or POCCCA antigen test strip. If results of microscopy and urine antigen testing were negative, patients who had positive results with urinalysis strip test were not treated because of the potential for false-positive results from any amount of menstrual bleeding in women of reproductive age. These patients were asked, however, to return for repeat testing. Children younger than 2 years and pregnant female patients were not treated because of a lack of praziquantel safety data in this population, but their contact information was recorded for follow-up. ${ }^{15}$ All other patients testing positive were treated with a single oral dose of praziquantel $(40 \mathrm{mg} /$ $\mathrm{kg}$ ) and asked to return in 8 weeks for repeat testing.

\section{RESULTS}

\section{STUDY PARTICIPANTS}

In total, 500 patients were consented to participate in this study and provided a urine sample. Participant age ranged from 6 months to 80 years (female sex, $n=283$; male sex, $\mathrm{n}=217$ ). Of the 500 urine samples, 414 were observed with microscopy, 498 tested with urinalysis strip, and 494 tested with the POC-CCA assay, with 380 samples being analyzed by all three methods. Eighty-six patients were unable to provide sufficient urine $(>10 \mathrm{~mL})$ for microscopic observation.

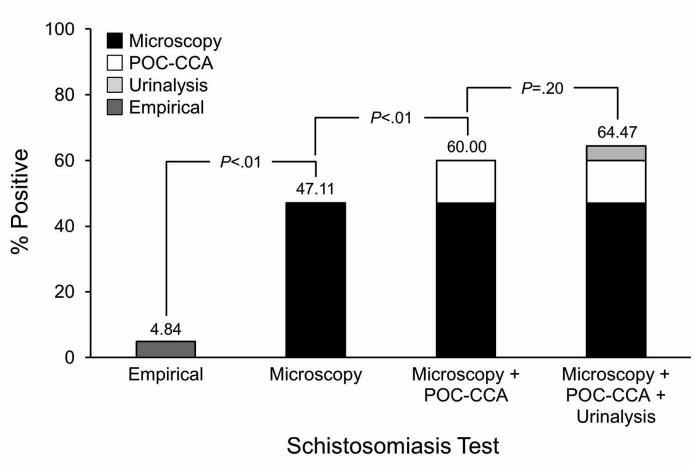

Figure 1. Positive results of schistosomiasis testing with microscopy, POC-CCA, and urinalysis.

Significance testing to examine the relation between schistosomiasis detection method and positive schistosomiasis screening result was performed with $\chi^{2}$ tests of independence ( $P<0.05$ defined as statistical significance). POC-CCA indicates point-of-care circulating cathodic antigen.

\section{COMPARISON OF SCREENING METHODS FOR SCHISTOSOMIASIS}

Before the present study, empirical diagnosis based on patient report of gross hematuria yielded a diagnosis rate of 4.84\% (Figure 1). With use of centrifuge microscopy during this study, a larger proportion of patients received the diagnosis of schistosomiasis $(P<0.01)$. To additionally identify patients infected with $S$. mansoni for whom eggs were not detectable with urine microscopy, we performed additional testing using the POC-CCA test for detection of antigens of $S$. haematobium and $S$. mansoni, resulting in a further increase in diagnosis rate $(P<0.01)$. Because of the low sensitivity of urine microscopy for detection of $S$. haematobium, additional urinalysis strip testing was added to identify potentially infected patients who may be missed with microscopy observation. The addition of urinalysis strip testing increased the presumptive schistosomiasis diagnosis rate; yet, this increase was not statistically significant.

\section{ASSESSMENT OF URINE TESTING METHODS FOR DETECTION OF S. HAEMATOBIUM INFECTION}

Samples from 380 study participants were tested with all 3 main methods, including the standard visualization of eggs with urine centrifuge microscopy. From these tests, 180 patients were confirmed positive for S. haematobium infection through visualization of eggs with urine centrifuge microscopy. This result enabled the assessment of the sensitivity of $S$. haematobium detection for urinalysis strip testing by comparison with these true positives. Urinalysis testing yielded a sensitivity of $77.65 \%$. Additionally, $67.03 \%$ of the positive urine microscopy samples also tested positive with the POC-CCA test.

\section{COST OF SCREENING FOR SCHISTOSOMIASIS}

The total cost to perform centrifuge microscopy, including 
Table 1. Costs of screening methods for schistosomiasis

\begin{tabular}{|c|c|c|c|c|c|c|c|}
\hline \multirow[b]{2}{*}{ Test } & \multirow[b]{2}{*}{$\begin{array}{l}\text { Labor } \\
\text { time } \\
\text { required, }{ }^{a} \\
\text { min }\end{array}$} & \multicolumn{6}{|c|}{ Cost, US\$ } \\
\hline & & Labor & $\begin{array}{c}\text { Disposable } \\
\text { materials }\end{array}$ & Investment & $\begin{array}{l}\text { Total to } \\
\text { screen } \\
100 \\
\text { patients }\end{array}$ & $\begin{array}{l}\text { Total to } \\
\text { screen } \\
1,000 \\
\text { patients }\end{array}$ & $\begin{array}{c}\text { Total to } \\
\text { screen } \\
10,000 \\
\text { patients }\end{array}$ \\
\hline $\begin{array}{l}\text { Centrifuge } \\
\text { microscopy }\end{array}$ & 10 & 0.047 & 0.02 & 380 & 386.70 & 447 & 1,050 \\
\hline $\begin{array}{l}\text { Gravity } \\
\text { microscopy }\end{array}$ & 7.5 & 0.031 & 0.02 & 300 & 305.10 & 351 & 810 \\
\hline $\begin{array}{l}\text { POC-CCA } \\
\text { Schistosoma } \\
\text { mansoni urine } \\
\text { test }\end{array}$ & 1.5 & 0.006 & 2.00 & 0 & 200.60 & 2,006 & 20,060 \\
\hline Urinalysis & 1 & 0.004 & 0.14 & 0 & 14.40 & 144 & 1,440 \\
\hline
\end{tabular}

Abbreviation: POC-CCA, point-of-care circulating cathodic antigen.

${ }^{a}$ Labor time included time to centrifuge samples, place urine in a tube, pipette samples, observe the microscope, submerge tests in urine, and read results. It also included time to clean reusable materials, done in accordance with Malagasy standards: 1) initial cleaning with hot boiled water, 2) cleaning with bleach, 3) cleaning with soap and water, and 4) a second round with hot boiled water. Labor costs were evaluated on the basis of the hourly wage of the nurse practitioner at Mada Clinics, who earned US $\$ 0.25$ per hour or US $\$ 2.00$ per day.

parts, labor, and time to sterilize reusable equipment, was US\$0.07 per test, with an additional US\$380 upfront investment in equipment (Table 1 ). The cost to perform gravity microscopy, including parts, labor, and time to sterilize reusable equipment, was US\$0.05 per test, with an additional US $\$ 300$ upfront investment in equipment. In contrast to microscopy, POC-CCA $S$. mansoni urine testing and urinalysis strip testing required no investment in equipment. Per test, a POC-CCA urine test cost US $\$ 2.00$ and a urinalysis strip test cost US\$0.14.

\section{CENTRIFUGATION VERSUS GRAVITY SEDIMENTATION FOR MICROSCOPY SAMPLE PREPARATION}

Centrifugation is the accepted standard of sample processing for detection of $S$. haematobium infection with urine microscopy. Gravity sedimentation is a lower-cost alternative that may be easier to implement in a low-resource setting. $\chi^{2}$ analysis was performed to examine the relation between these detection methods and positive schistosomiasis screening results. No difference was observed between these sample preparation methods $(P=0.49)$.

\section{PREVALENCE TRENDS AND GEOGRAPHIC DISTRIBUTION}

To understand the demographic characteristics of schistosomiasis in the region, we recorded the village of residence and age of all participants. Age categories were those used on Malagasy Ministry of Health forms. The greatest proportion of patients testing positive for schistosomiasis was observed in the 10-to-24-year age category (72.79\%); the lowest percentage was observed in the age category of 50 years and older (33.82\%) (Figure 2). $\chi^{2}$ analysis was performed to examine the relation between age category and positive schistosomiasis test result. Patients in the age category of 25 to 49 years were less likely than patients in the age category of 10 to 24 years to test positive for schistosomiasis $(P=0.005)$. Patients in the age category of 50 years and older

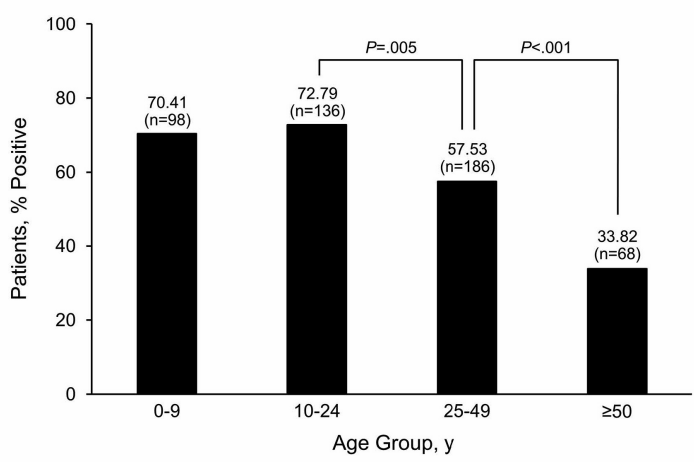

Figure 2. Age distribution of patients testing positive for schistosomiasis.

Positive infection was defined as visible eggs on microscopy, positive point-ofcare circulating cathodic antigen test result, or positive urinalysis test results of all male persons without an otherwise likely clinical explanation and all female persons younger than 8 years or older than 60 years without a likely clinical explanation. Significance testing to examine the relationship between age category and positive schistosomiasis screening result was performed with $\chi^{2}$ tests of independence $(P<0.05$ defined as statistical significance) .

were less likely than patients age 25 to 49 years to test positive for schistosomiasis $(P<0.001)$. The rate of schistosomiasis was reported for each of 6 villages where the majority (88.2\%) of participants resided (Figure 3 ).

\section{DISCUSSION}

Before this study, empirical diagnosis based on gross hematuria was the sole means to assess for schistosomiasis at Mada Clinics. Using several easily performed laboratory methods, we demonstrated that empirical diagnosis vastly 
underestimated the true prevalence of the disease. By comparing empirical diagnosis, gold-standard urine microscopy, POC-CCA testing, and urinalysis strip testing, we estimated the prevalence rate of schistosomiasis at $64.47 \%$ in the region served by Mada Clinics. This result is comparable with the 2010 countrywide estimation of schistosomiasis prevalence in Madagascar-52.21\%-and is higher than the $4.84 \%$ rate of diagnosis and treatment based on empirical methods previously used by Mada Clinics. ${ }^{6}$ This study showed the importance of using screening methods and diagnostic tests to detect and treat schistosomiasis in rural Madagascar.

Urine microscopy is the most specific test for $S$. haematobium detection and thus was used as an established goldstandard in this study. However, microscopy lacks high sensitivity because $S$. haematobium eggs may not pass each time an infected person urinates. Repeat testing on alternate days is recommended to increase overall testing sensitivity. However, the population in this study could not remain at the clinic, away from their work or family, for multiple days. To identify presumptively infected individuals missed in initial screening with urine microscopy, urinalysis strip testing was used to detect microhematuria, a potential product of the inflammation caused by $S$. haematobium.

Using urine microscopy as evidence of schistosomiasis, we found a $77.65 \%$ sensitivity of urinalysis strip testing for $S$. haematobium in the population, greater than the estimated sensitivity of 1 screen with urine microscopy (31\%) seen previously. ${ }^{16}$ In addition, meta-analysis has shown that specificity of urinalysis strip testing for S. haematobium approaches $97 \%$ in selected populations. ${ }^{17}$ Urinalysis testing is quick, requires little training or labor, and is cheaper than microscopy to implement when fewer than approximately 7,000 patients are screened.

$S$. mansoni is also present in rural northwestern Madagascar, its eggs passed primarily in stool. Neither urine microscopy nor urinalysis identifies patients infected with $S$. mansoni. Yet, antigens from this parasite can still enter urine and be detected with the POC-CCA test. Although definitive testing for $S$. mansoni has previously been achieved through the analysis of stool samples using the Kato-Katz smear, this study focused on urine-based assays due to the resource and practice limitations of the community. Additionally, the POC-CCA assay may cross-react with S. haematobium antigens, allowing for detection of both Schistosoma species through one test. ${ }^{12}$ A disadvantage of the cross-reactivity is that it does not allow for the differentiation of $S$. mansoni and $S$. haematobium infections in populations where both species are endemic. However, the treatment is the same for both infections. ${ }^{5}$

Urinalysis strip testing is initially the cheapest assay, thanks to the lack of necessary equipment. Yet, as the number of patients screened increases, both gravity and centrifuge microscopy become cheaper methods. Owing to the remarkable change in diagnosis rates brought by microscopy (4.84\% vs $47.11 \%)$, the staff at Mada Clinics has decided to hire and train a local employee in microscopy. A strategy to decrease the resources for microscopy is the use of gravity sedimentation in place of centrifugation, which we showed to produce comparable results while it avoided

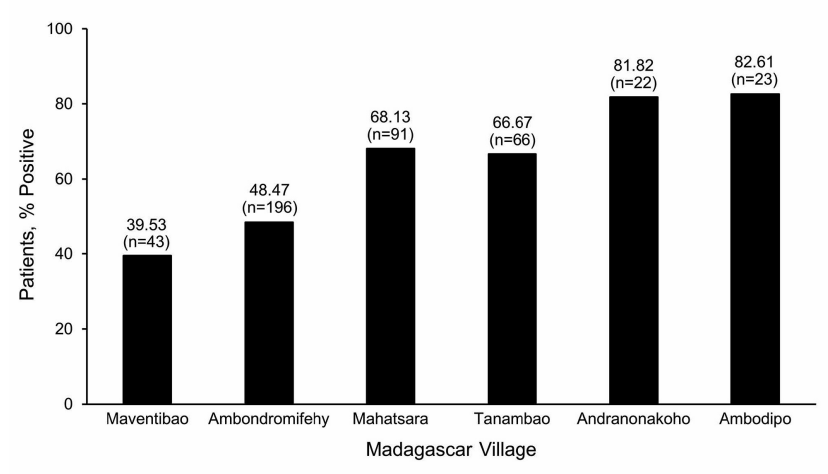

Figure 3. Geographic distribution of schistosomiasis infection.

\begin{abstract}
Positive infection was defined as visible eggs on microscopy, positive point-ofcare circulating cathodic antigen test result, or positive urinalysis test results in all male persons without an otherwise likely clinical explanation and all female persons younger than 8 years and older than 60 years without an otherwise likely clinical explanation. Maventibao is the village where Mada Clinics is located. Ambondromifehy is the next closest village to the clinic, and Mahatsara and Tanambao are approximately $7 \mathrm{~km}$ from the clinic. Andranonakoho and Ambodipo are the farthest 2 villages from the clinic in this dataset.
\end{abstract}

the cost and labor of a manual centrifuge. Nonetheless, centrifugation could deliver results within 20 minutes, while gravity filtration required 24 hours. The wait time required for gravity filtration was impractical for the patient population treated by Mada Clinics, some of whom traveled 40 $\mathrm{km}$ on foot. For this reason, Mada Clinics discontinued the use of gravity sedimentation following this study. This geographic limitation also precluded the assessment of specificity, which has previously been achieved by screening negative patients separate times to establish a true negative diagnosis.

Despite the high sensitivity and high specificity (established false positive rate of $<5 \%$ ) of the POC-CCA test, as well as ease of use, Mada Clinics discontinued its use because of excessive cost. ${ }^{12}$ Although Kato-Katz thick smear would be a less costly alternative to screen for $S$. mansoni, it requires additional investment into sample collection, processing, and analysis infrastructure. ${ }^{18}$ In the current absence of a permanent microscopist, the staff at Mada Clinics has decided to use urinalysis strip testing to screen most patients for schistosomiasis. This decision was made by carefully balancing costs against the monthly budget of the clinic and the time constraints of employees.

When we analyzed patient demographic characteristics, we observed a significant decrease in schistosomiasis rate as the category age increased. Previous studies have indicated that an age-related increase in immunity to schistosomiasis may exist in populations who live in endemic areas. ${ }^{19}$ In addition, it has been hypothesized that older individuals interact with infected water sources less frequently and that accumulated scar tissue may prevent eggs from entering the urinary tract. ${ }^{20}$ However, the study population tended to use the same water sources for bathing, washing clothes, and washing kitchen utensils regardless of 
age, suggestive that increased immunity may have a dominant role.

Considerable heterogeneity was observed in the rate of schistosomiasis by village of residence. The reasons for this heterogeneity warrant further investigation, particularly in high-prevalence villages, including Andrononakoho and Ambodipo. We noted that no village in this study had public latrines and all villages practiced open defecation, which presents an opportunity for future sanitation efforts to lower the observed rates.

\section{CONCLUSIONS}

In summary, we implemented an affordable schistosomiasis screening program that showed improved ability to detect infection compared with empirical methods previously in place. Our assessment of schistosomiasis screening tools provides a model that could be implemented in low-income countries for the diagnosis and surveillance of schistosomiasis on a national scale. Importantly, all screening methods used in this study can be performed in the field in a noninvasive manner with urine samples and without need for refrigeration.

To our knowledge, this is the first study of schistosomiasis prevalence in this region of Madagascar, the results of which stirred local communities to install multiple infrastructure projects aimed at improving access to potable water. Thus, we demonstrated benefits of schistosomiasis screening that extend beyond the treatment of infected individuals and can inform future public health efforts. Greater than one-half of the Madagascar population may be infected with schistosomiasis, underscoring the importance of effective low-cost methods for schistosomiasis detection, treatment, and prevention.

\section{ETHICS APPROVAL AND INFORMED CONSENT}

This study was approved by the Mayo Clinic Institutional Review Board in Rochester, Minnesota, and the Malagasy Ministry of Health in Antsiranana. For all participants in this study verbal consent was given by patients before study enrollment, including parental consent for minors.

\section{FUNDING}

This study was funded by the nonprofit organization Mada Clinics, Inc.

\section{AUTHORSHIP CONTRIBUTIONS}

Study conception and design (KER). Data collection (KER, ML, AMS, MF). Clinical input and study supervision (BSP, LMB, CRL). Data analysis and drafting of the manuscript (KER, EPG).

\section{COMPETING INTERESTS}

The authors completed the Unified Competing Interest form at www.icmje.org/coi_disclosure.pdf (available upon request from the corresponding author), and declare no conflicts of interest.

Submitted: February 02, 2021 GMT, Accepted: March 16, 2021 GMT 


\section{REFERENCES}

1. World Health Organization. Schistosomiasis. Published 2020. Accessed February 17, 2020. https://w ww.who.int/news-room/fact-sheets/detail/schistosom iasis

2. Centers for Disease Control and Prevention, US Department of Health \& Human Services. Neglected tropical diseases. Published 2019. Accessed February 17, 2020. https://www.cdc.gov/globalhealth/ntd/disea ses/index.html

3. Thetiot-Laurent SA, Boissier J, Robert A, Meunier B. Schistosomiasis chemotherapy. Angew Chem Int Ed Engl. 2013;52(31):7936-7956. doi:10.1002/anie.20120 $\underline{8390}$

4. Training in Tropical Diseases, United Nation's Children Fund, United Nations Development Programme, World Health Organization. Praziquantel: getting the dosage right. Published 2008. Accessed February 17, 2020. https://www.who.i nt/tdr/news/2008/praziquantel-dosing/en/

5. Vale N, Gouveia MJ, Rinaldi G, Brindley PJ, Gartner F, Correia da Costa JM. Praziquantel for schistosomiasis: single-drug metabolism revisited, mode of action, and resistance. Antimicrob Agents Chemother. 2017;61(5). doi:10.1128/AAC.02582-16

6. Rollinson D, Knopp S, Levitz S, et al. Time to set the agenda for schistosomiasis elimination. Acta Trop. 2013;128(2):423-440. doi:10.1016/j.actatropic a.2012.04.013

7. Index Mundi. Madagascar: people practicing open defecation (\% of population). Accessed February 18, 2020. https://www.indexmundi.com/facts/madagasca r/indicator/SH.STA.ODFC.ZS

8. World Bank Group. Madagascar. Published 2019. Accessed February 18, 2020. https://data.worldbank.o $\mathrm{rg} /$ country/madagascar

9. Sawyer SG. Schistosomiasis (Bilharzia) in Madagascar: a case study of a neglected tropic disease. Published 2013. Accessed February 18, 2020. https://digitalcollections.sit.edu/isp_collection/1675/

10. Expanded Special Project for Elimination of Neglected Tropical Diseases (ESPEN), World Health Organization. Madagascar. Accessed February 18, 2020. http://espen.afro.who.int/countries/madagascar

11. Gray DJ, Ross AG, Li YS, McManus DP. Diagnosis and management of schistosomiasis. BMJ. 2011;342:d2651. doi:10.1136/bmj.d2651
12. Barenbold O, Garba A, Colley DG, et al. Translating preventive chemotherapy prevalence thresholds for Schistosoma mansoni from the KatoKatz technique into the point-of-care circulating cathodic antigen diagnostic test. PLoS Negl Trop Dis. 2018;12(12):e0006941. doi:10.1371/journal.pntd.0006 941

13. Parikh R, Mathai A, Parikh S, Chandra Sekhar G, Thomas R. Understanding and using sensitivity, specificity and predictive values. Indian J Ophthalmol. 2008;56(1):45-50. doi:10.4103/0301-4738.37595

14. Koukounari A, Webster JP, Donnelly CA, et al. Sensitivities and specificities of diagnostic tests and infection prevalence of Schistosoma haematobium estimated from data on adults in villages northwest of Accra, Ghana. Am J Trop Med Hyg.

2009;80(3):435-441.

15. Centers for Disease Control and Prevention, US Department of Health \& Human Services. Parasites: schistosomiasis. Resources for health professionals. Published 2018. Accessed February 18, 2020. http s://www.cdc.gov/parasites/schistosomiasis/health_pr ofessionals/index.html

16. Vinkeles Melchers NV, van Dam GJ, Shaproski D, et al. Diagnostic performance of schistosoma realtime PCR in urine samples from Kenyan children infected with Schistosoma haematobium: day-to-day variation and follow-up after praziquantel treatment. PLoS Negl Trop Dis. 2014;8(4):e2807. doi:10.1371/jour nal.pntd.0002807

17. King $\mathrm{CH}$, Bertsch D. Meta-analysis of urine heme dipstick diagnosis of Schistosoma haematobium infection, including low-prevalence and previouslytreated populations. PLoS Negl Trop Dis. 2013;7(9):e2431. doi:10.1371/journal.pntd.0002431

18. Barenbold O, Raso G, Coulibaly JT, N'Goran EK, Utzinger J, Vounatsou P. Estimating sensitivity of the Kato-Katz technique for the diagnosis of Schistosoma mansoni and hookworm in relation to infection intensity. PLoS Negl Trop Dis. 2017;11(10):e0005953. doi:10.1371/journal.pntd.0005953

19. Colley DG, Secor WE. Immunology of human schistosomiasis. Parasite Immunol. 2014;36(8):347-357. doi:10.1111/pim.12087

20. Pinot de Moira A, Fulford AJ, Kabatereine NB, Ouma JH, Booth M, Dunne DW. Analysis of complex patterns of human exposure and immunity to Schistosomiasis mansoni: the influence of age, sex, ethnicity and IgE. PLoS Negl Trop Dis. 2010;4(9). doi:1 0.1371/journal.pntd.0000820 